\title{
Análise de transmissão de preços do mercado atacadista de melão do Brasil
}

\author{
*Rodrigo de Oliveira Mayorga \\ **Ahmad Saeed Khan \\ ***Ruben Dario Mayorga \\ ****Patrícia Verônica Pinheiro Sales Lima \\ $* * * * *$ Mario Antônio Margarido
}

Resumo: O presente estudo se propôs a analisar a relação existente entre os mercados atacadistas de melão amarelo no Brasil. Para analisar a transmissão de preços entre estes mercados, utilizaram-se métodos de séries de tempo, teste de raiz unitária, teste de co-integração de Johansen, o modelo Auto-regressivo Vetorial (VAR), decomposição da variância dos erros de previsão e função resposta ao impulso. O período analisado abrangeu janeiro de 2001 a dezembro de 2005. Os resultados mostraram que, apesar dos pólos Açu/Mossoró-RN e Baixo JaguaribeCE representarem as maiores áreas produtoras nacionais de melão, variações nos preços de atacado de melão amarelo das Centrais de Abastecimento de Natal e Fortaleza, não afetam de maneira significativa os preços dos outros mercados estudados. No entanto, variações nos preços da Companhia de Entrepostos e Armazéns Gerais de São Paulo

\footnotetext{
* Mestre em Economia Rural pelo Programa de Pós-Graduação em Economia Rural da Universidade Federal do Ceará. rsmyg@yahoo.com.br

**PhD em Economia Agrícola. Professor Titular do Departamento de Economia Agrícola da Universidade Federal do Ceará. Bolsista CNPq. saeed@ufc.br

***PhD em Ciência dos Recursos de Terras Áridas. University of Arizona, U.A. Professor do Departamento de Economia Agrícola da Universidade Federal do Ceará. dario@ufc.br ****Doutora em Economia Aplicada. Professora adjunta do Departamento de Economia Agrícola da Universidade Federal do Ceará. pvpslima@gmail.com *****Doutor em Economia Aplicada. Pesquisador do Instituto de Economia Agrícola de São Paulo.mamargarido@iea.sp.gov.br
} 
(CEAGESP) têm impacto sobre todos os mercados atacadistas analisados. Assim sendo, a CEAGESP, representando os atacadistas nacionais, e sendo a maior intermediadora do país, comanda o mercado brasileiro de melão amarelo comercializado, constituindo, na prática, um mercado oligopolístico.

Palavras-chave: Mercado atacadista, transmissão de preços, modelo VAR.

\section{Classificação JEL: Q13}

Abstract: The present study has the purpose to analyze the relationship among wholesale market of yellow melon in Brazil. Time series methods, unit root test, Johansen cointegration test, Vectorial Autoregressive models (VAR), variance decomposition of prediction error and impulse response function were used to analyze the price transmission among these markets. The analyzed period embraces January 2001 to December 2005. The results showed that, despite Açu/Mossoró-RN and Baixo Jaguaribe-CE represents the national biggest melon production areas, wholesale prices deviations from the Supply Centers of Natal and Fortaleza, didn't affect in a significant way the other markets studied. On the other hand, price variation of the CEAGESP (Companhia de Entrepostos e Armazéns Gerais de São Paulo), affects all the others wholesale markets analyzed. Therefore, the CEAGESP, representing national wholesalers, it being the biggest intermediary of the country, commands the Brazilian yellow melon market, establishing, in practice, an oligopolistic market.

Key words: Wholesale market, price transmission, VAR model.

\section{JEL classification: Q13}

\section{Introdução}

O meloeiro (Cucumis melo L.) é uma olerícola muito apreciada e de popularidade ascendente no Brasil. O fruto, rico em vitaminas $A, B, B 2$, B5 e C, sais minerais - como potássio, sódio e fósforo - apresenta valor energético relativamente baixo (20 a $62 \mathrm{kcal} / 100 \mathrm{~g}$ de polpa), é consumi- 
do in natura ou na forma de suco. Atribui-se, ainda, ao fruto maduro do melão propriedades terapêuticas, diuréticas, calmantes, mineralizantes e alcalizantes (FRUTAS DO BRASIL, 2003, p.13).

Dentre os fatores que determinam a qualidade do melão, destacamse a eficiência na irrigação, o manejo fitossanitário, nutricional e póscolheita, o tipo de transporte e outros custos de transação, especialmente referentes à elaboração dos contratos com as devidas salvaguardas de proteção aos produtores. Na verdade, a qualidade dos melões está associada à tecnologia utilizada pelas grandes empresas produtoras e compradoras de produto.

De acordo com os dados do AGRIANUAL (2005), o melão é cultivado principalmente no Nordeste ( $94,8 \%$ da produção total), com destaque para os estados do Rio Grande do Norte e Ceará, que respondem, respectivamente, por $51,6 \%$ e $26,1 \%$ da produção brasileira. Os agropólos irrigados do Açu/Mossoró-RN e do Baixo Jaguaribe-CE são os centros produtores de maior expressão e têm produtividade média de 28,3 t/ha e 24,9 t/ha, respectivamente. O outro importante pólo meloeiro do País é PE/BA, que fica assentado no agropólo do submédio São Francisco, abrangendo Pernambuco e Bahia, produzindo aproximadamente 17\% da produção nacional. Além destes agropólos, outros estados brasileiros produzem melão: Rio Grande do Sul, São Paulo, Paraná, Santa Catarina e Mato Grosso.

O melão do tipo amarelo é o mais resistente às contingências do transporte de longas distâncias e ao armazenamento em temperatura ambiente, o que explica a preferência de cultivo por este tipo de melão pelos produtores. A área plantada alcança $70 \%$ da área cultivada com este tipo de melão (FRUTISÉRIES, 2003, p. 2).

Por trás do comportamento dos preços no mercado interno e externo, das commodities, como é o caso do melão amarelo no Brasil, existem questões estruturais de mercado, características específicas de produção, comercialização e consumo que é preciso conhecer para obter compreensão mais apurada do relacionamento dos preços do mercado brasileiro do melão amarelo, e suas implicações.

Conhecer o relacionamento entre os preços dos mercados atacadistas de frutas no Brasil pode ser um instrumental na busca da melhor compreensão do comportamento desses mercados, especificamente 
para os melões amarelos, que apresentam crescimento na produção, comercialização e consumo em todo o Brasil.

\section{Objetivos}

\section{Objetivo Geral}

Examinar a relação de transmissão de preços e causalidade entre os mercados atacadistas de melão amarelo no Brasil.

\section{Objetivos Específicos}

Analisar se as maiores regiões produtoras, Ceará e Rio Grande do Norte, têm alguma relação de causalidade com os mercados atacadistas analisados;

Verificar de que forma estão relacionados os mercados atacadistas de melão amarelo no Brasil; e,

Analisar se os mercados analisados são integrados.

\section{Referencial teórico}

\subsection{Modelos Estáticos}

Modelos de natureza estática representam a hipótese implícita de que todos os ajustamentos devem ocorrer instantaneamente, ou seja, não há defasagens distribuídas ao longo do tempo.

Os primeiros pesquisadores do setor agrícola a estudar transmissão de preços e integração de mercado utilizaram, em sua maioria, análise de correlação de preços e regressão simples. Estes modelos, no entanto, passaram a ser criticados pela negligência que mascara a presença de outros fatores que podem causar variações nos preços, como inflação de preços, sazonalidade (principalmente na agricultura), crescimento populacional, problemas climáticos entre outros (TIMMER, 1974; HARRIS, 1979 apud GOLETTI; RAISUDDIN; FARID, 1995, p. 188). Além disso, não havia o cuidado de verificar se as séries eram estacionárias.

Uma maneira de evitar estas críticas foi a de considerar a diferenciação de preços, que tem a propriedade atrativa de interpretar integração de mercado como interdependência de mudança de preços em diferen- 
tes mercados. Além disso, diferenciação de preço elimina a tendência comum que causa regressão espúria (GOLETTI; RAISUDDIN; FARID, 1995, p. 188). Nesse caso, as estatísticas de avaliação $|t|, F$ e $R^{2}$, apesar de apresentarem valores elevados, podem não traduzir a verdadeira relação teórica entre as variáveis.

Apesar da estacionariedade poder ser alcançada pela diferenciação, nem sempre é uma solução satisfatória (PLOSSER; SCHWERT, 1978 apud ARDENI, 1989, p. 661). Além disso, em muitos casos, o que importa são as relações entre as variáveis em nível, o que seria perdido se as séries fossem diferenciadas.

Outra crítica à maior parte destes modelos é a sua natureza estática e a omissão de defasagens distribuídas permitindo que ajustamentos ao longo do tempo sejam estabelecidos (COELHO, 2002, p. 46).

\subsection{Modelos Dinâmicos}

Freqüentemente não é suficiente dizer que mercados são integrados. Procura-se conhecer a extensão da integração. Um modelo dinâmico tem a vantagem de que o pesquisador pode distinguir entre os conceitos de integração de mercado instantânea (impacto imediato de choques de preços) e a idéia menos restritiva de integração, isto é, processos de ajustamento dinâmico de longo prazo (impactos que são formados em um lapso de tempo). Os modelos de defasagem distribuída são capazes de captar esse efeito multiplicador.

Uma categoria de modelos dinâmicos muito usada na análise de transmissão de preços envolve o conceito de causalidade. Os trabalhos de Granger (1969) e de Sims (1972) introduziram e popularizaram a aplicação de testes de causalidade para pesquisas em Economia Agrícola e vários outros campos (ZAPATA; GIL, 1999, p. 2).

Bishop (1979) descreve as duas metodologias de causalidade para análise de preços agrícolas, no que foi sugerido por uma série de autores em todo o mundo, inclusive no Brasil.

Outra estrutura de modelo dinâmico foi proposta por Ravallion (1986). Em seu artigo ele utiliza vetores auto-regressivos ${ }^{1}$ para analisar

${ }^{1}$ O Modelo de Vetores Auto-regressivos foi introduzido como uma alternativa aos modelos de equações múltiplas pelo trabalho de Sims. C. A. Sims, "Macroeconomics and 
a relação entre os preços dos mercados agrícolas de Bangladesh, durante o período de 1972-1975, marcados pela fome naquele país.

Muitas séries temporais parecem seguir um passeio aleatório, e isso sugere que em geral se deve diferenciar uma variável antes de usá-la em uma regressão. Ainda que isto seja aceitável, a diferenciação pode resultar em perda de informações valiosas sobre a relação de longo prazo que um modelo econométrico procura exprimir e que são indicados por variáveis em níveis de acordo com a teoria.

Segundo Pindyck e Rubinfeld (2004, p. 590), há situações em que se pode estimar uma regressão entre duas variáveis, ainda que sejam passeios aleatórios. Diz-se, neste caso, que as variáveis são co-integradas, havendo uma combinação linear entre elas, que são estacionárias.

A idéia de co-integração é verificar se duas ou mais séries analisadas são integradas de mesma ordem, e, neste caso, verificar se existe uma combinação linear entre elas, que seja estacionária.

\section{Material e métodos}

\section{1. Área Geográfica de Estudo e Origem dos Dados}

Os dados utilizados neste trabalho foram obtidos na Secretaria da Agricultura e Pecuária, Ceará (SEAGRI). Consistem em séries de preços semanais de melão amarelo no período de janeiro de 2001 a dezembro de 2005 e correspondem aos mercados atacadistas de São Paulo-SP, Natal-RN, Fortaleza-CE, Salvador-BA, Recife-PE, CuritibaPR, Belo Horizonte-MG e Brasília-DF. Os dados estão expressos em reais por quilo $(\mathrm{R} \$ / \mathrm{kg})$.

Algumas considerações devem ser feitas no que respeita à deflação de preços e ao deflator a ser utilizado. De acordo com Pino e Rocha (1994 apud MARGARIDO, 1998, p. 71), sendo $z_{t}$ a série original não deflacionada e $d_{t}$ um deflator apropriado tal que:

$$
y_{t}=\frac{z_{t}}{d_{t}}
$$

então,

Reality”, Econometric, vol. 48,1980, pp. 1-48. 


$$
Y_{t}=\log \frac{y_{t}}{y_{t-1}}=\log \frac{z_{t} / d_{t}}{z_{t-1} / d_{t-1}}=\log \frac{z_{t}}{z_{t-1}}-\log \frac{d_{t}}{d_{t-1}}
$$

Por outro lado, é usual ajustar o modelo à série centrada ao redor da média quando se toma uma diferença de ordem 1 (um): $Y_{t}-\bar{Y}$. Neste caso,

$$
\begin{aligned}
& \bar{Y}=\frac{1}{n} \sum_{i} Y_{i} \\
& =\frac{1}{n} \sum\left(\log \frac{z_{t}}{z_{t-1}}-\log \frac{d_{t}}{d_{t-1}}\right) \\
& =\frac{1}{n} \sum \log \frac{z_{t}}{z_{t-1}}-\frac{1}{n} \sum \log \frac{d_{t}}{d_{t-1}}
\end{aligned}
$$

Porém, se a taxa de inflação for semelhante de mês a mês no período considerado, então, qualquer que seja $t$, essa relação é aproximadamente constante. Logo,

$$
\begin{gathered}
\frac{d_{t}}{d_{t-1}} \approx k \\
\log \frac{y_{t}}{y_{t-1}}-\frac{1}{n} \sum \log \frac{y_{i}}{y_{i-1}}=Y_{t}-\bar{Y} \\
=\log \frac{z_{t}}{z_{t-1}}-\log \frac{d_{t}}{d_{i-1}}-\frac{1}{n} \sum \log \frac{z_{i}}{z_{i-1}}+\frac{1}{n} \sum \log \frac{d_{i}}{d_{i-1}} \\
\approx \log \frac{z_{t}}{z_{t-1}}-\frac{1}{n} \sum \log \frac{z_{i}}{z_{i-1}}
\end{gathered}
$$

Portanto, da forma como as séries foram transformadas, o modelo com a série deflacionada é, aproximadamente, equivalente ao modelo com a série sem deflação. No presente trabalho, foi empregada a transformação logarítmica. Utilizou-se no presente estudo o programa econométrico Eviews 5.0. 
As séries estimadas para análise de relação de preços de melão no mercado atacadista brasileiro foram, então: LSP - logaritmo natural do preço de São Paulo-SP; LNAT - logaritmo natural do preço de Natal-RN; LFOR - logaritmo natural do preço de Fortaleza-CE; LSAL - logaritmo natural do preço de Salvador-BH; LREC - logaritmo natural do preço de Recife-PE; LCUR - logaritmo natural do preço de Curitiba-PR; LBH - logaritmo natural do preço de Belo Horizonte-MG; e LBRA - logaritmo natural do preço de Brasília-DF.

\subsection{Verificação de Estacionariedade}

\subsubsection{Função Autocorrelação e o Correlograma}

Uma das principais ferramentas utilizadas para detectar estacionariedade é o teste de função de autocorrelação (FAC) e o correlograma resultante, que é simplesmente a representação gráfica da FAC contra o tamanho de defasagens.

Na prática, é necessário trabalhar com o coeficiente de autocorrelação amostral. Logo, a função de autocorrelação amostral na defasagem $k$ é:

$$
\hat{\rho}_{k}=\frac{\hat{\gamma}_{k}}{\hat{\gamma}_{0}}
$$

que é simplesmente a razão entre a covariância amostral e a variância amostral. Quanto mais $\hat{\rho}_{k}$ aproxima-se de 1 , maior o indício de que a série temporal é não estacionária. Outro indicador de não-estacionariedade é a representação gráfica de $\hat{\rho}_{k}$, conhecida como correlograma amostral. Quando o correlograma inicia com um valor bem alto (próximo de 1) e decai gradativamente, tem-se forte indício de série temporal não estacionária (GUJARATI, 2000, p. 721).

\subsubsection{Teste de Raiz Unitária}

Para testar a presença ou não de raiz unitária na série e ordem de integração, foi usado o Teste de Dickey-Fuller Aumentado - ADF, desenvolvido por Dickey e Fuller (1981), que tem como base a seguinte expressão: 


$$
\Delta Y_{t}=\beta+\delta T+\gamma Y_{t-1}+\alpha_{i} \sum_{i=1}^{m} \Delta Y_{t-i}+\varepsilon_{t}
$$

em que $\beta$ é uma constante (ou intercepto); $\delta t$ é a tendência; $\Delta$ é o operador diferença, o qual é representado como: $\Delta_{t}=Y_{t}-Y_{t-1}$.

$\mathrm{O}$ teste de ADF é, entretanto, muito sensível à presença de valores atípicos (FRANSES; HALDRUP, 1994; CATI; GARCIA; PERRON, 1999 apud FERNANDES; TORO, 2005, p. 9). Neste caso, utiliza-se o teste KPSS desenvolvido por Kwiatkowski, et al. (1992).

Estes autores sugeriram um teste cuja principal característica é a inversão das hipóteses em teste, isto é, a hipótese nula assume a noção de que a série é estacionária e sob a hipótese alternativa a série é integrada de ordem um. A vantagem deste teste resulta da falta de potência dos testes convencionais, isto é, tendem a não rejeitar a hipótese nula com demasiada freqüência.

Seja $y_{t}, t=1,2, \ldots, T$, a série observada para a qual quer se testar a estacionariedade. Assumindo a noção de que possa se decompor a série na soma de tendência determinística, passeio aleatório, e erro estacionário:

$$
y_{t}=\xi_{t}+r_{t}+\varepsilon_{t}
$$

Em (3.6) $r_{t}$ é um passeio aleatório:

$$
r_{t}=r_{t-1}+u_{t}
$$

em que $u_{t}$ iid $\left(0, \sigma_{u}^{2}\right)$, processo independente e identicamente distribuído, isto é, distribuição normal com média zero e variância constante. O valor inicial de $r_{0}$ é considerado fixo e tem função de intercept o. A hipótese de estacionariedade é simplesmente $\sigma_{u}^{2}=0$. Desde que, assumindo $\varepsilon_{t}$ estacionário, sob a hipótese nula de $y_{t}$ ser estacionário em torno de uma tendência. Também considera-se um caso especial do modelo (3.6) no qual considera $\xi=0$, sob a hipótese nula $y_{t}$ ser estacionário em torno de um nível $\left(r_{0}\right)$, em vez de uma tendência.

A estatística utilizada é teste unilateral LM para hipótese de $\sigma_{u}^{2}=0$, sob uma pressuposição mais forte de que $u_{t}$ é normal e que $\varepsilon_{t}$ é iid $\mathrm{N}\left(0, \sigma_{u}^{2}\right)$.

Os valores críticos para testar LM são baseados nos resultados assintóticos tabelados por KPSS.

De acordo com Kwiatkowski et al. (1992, p. 176), o teste KPSS tende a 
complementar o testes de raiz unitária, como o teste de Dickey-Fuller. Testando ambas as hipóteses, de raiz unitária e de estacionariedade, podem-se distinguir séries que aparentam ser estacionárias, séries que aparentam possuir raiz unitária e para as quais os dados (ou testes) não são suficientemente informativos para assegurar se são estacionárias ou integradas.

\subsection{Vetores Auto-regressivos (VAR)}

Para simplificar a análise será utilizado um exemplo de sistema de equações com duas variáveis, as quais se assume sejam interdependentes e também relacionadas por uma memória auto-regressiva, isto é, a seqüência $X_{t}$ é afetada pelo seu passado e pela seqüência $Z_{t}$ e vice-versa. A estacionariedade é uma condição fundamental para as propriedades dos estimadores do modelo. Analiticamente, pode-se representar o VAR:

$$
\begin{aligned}
& X_{t}=\alpha_{10}+\alpha_{11} X_{t-1}+\alpha_{12} Z_{t-1}+\varepsilon_{t 1} \\
& Z_{t}=\alpha_{20}+\alpha_{21} X_{t-1}+\alpha_{22} Z_{t-1}+\varepsilon_{t 2}
\end{aligned}
$$

Pode-se escrever o modelo VAR em notação matricial, como:

$$
Y_{t}=\alpha+\Pi_{1} Y_{t-1}+\Pi_{2} Y_{t-2}+\ldots+\Pi_{p} Y_{t-p}+\varepsilon_{t}
$$

em que

$Y_{t}$ : vetor $(n \times 1)$ auto-regressivo de ordem $p$;

$\alpha_{0}$ : vetor ( $\left.n \times 1\right)$ de interceptos;

$\Pi_{i}$ : matriz de parâmetros de ordem $(n \times n)$;

$\varepsilon_{t}$ : termo de erro $\varepsilon_{t} \sim N(0, \Omega)$.

Esses coeficientes não levam em conta os relacionamentos entre as variáveis expressas no modelo VAR. Então, o caminho mais apropriado para avaliar os impactos das inovações é dado pela função impulso - resposta.

\subsubsection{Função de Impulso-Resposta}

Uma função impulso-resposta delineia o comportamento das séries 
incluídas no modelo VAR em resposta a choques ou mudanças provocadas por variáveis residuais.

A simulação baseada na função impulso-resposta do VAR provê um mecanismo para estimar respostas a choques, sem manter a pressuposição de condições, ceteris paribus, para outras variáveis do modelo.

Considerando-se o modelo VAR (3.8) e (3.9), o efeito de um choque, ou de uma mudança em, $\varepsilon_{t 1}$, altera imediatamente os valores correntes da variável $X_{t}$, mas também os valores futuros de $X_{t}$ e $Z_{t}$, uma vez que os valores defasados $X_{t-1}$ aparecem nas duas equações.

Raciocínio análogo pode ser aplicado às demais inovações. Se as inovações $\varepsilon_{t 1}$ e $\varepsilon_{t 2}$ não fossem correlacionadas contemporaneamente, a interpretação da função impulso-resposta seria direta e cada uma delas diretamente associada a uma variável, como aparece no modelo. Nesse caso, a função impulso-resposta relativa à inovação $\varepsilon_{t 2}$, por exemplo, mediria o efeito de um choque sobre os valores correntes e futuros de $Z_{t}$ e sobre os valores futuros de $X_{t}$.

\subsubsection{Decomposição da Variância}

De acordo com Enders (1995), a decomposição de variância fornece o percentual do erro da variância prevista atribuída aos choques de uma determinada variável versus os choques nas outras variáveis do sistema. Se os choques observados numa variável $z$ não são capazes de explicar a variância do erro de previsão da variável $y$, diz-se que a seqüência $y$ é exógena. Caso contrário, diz-se que a seqüência é endógena.

A decomposição da variância dos erros de previsão mostra a evolução do comportamento dinâmico apresentado pelas variáveis do sistema econômico, ao longo do tempo, isto é, permite separar a variância dos erros de previsão para cada variável em componentes que podem ser atribuídos por ela própria e pelas demais variáveis endógenas, isoladamente, apresentando, em termos percentuais, qual o efeito que um choque não antecipado sobre determinada variável tem sobre ela própria e as demais variáveis pertencentes ao sistema (MARGARIDO et al., 2002, p. 78). 


\subsection{Vetor de Correção de Erros (VEC)}

A condição necessária para que os estimadores obtidos possuam propriedades desejáveis é que as variáveis do VAR sejam estacionárias. Caso contrário, a existência de raízes unitárias deve ser levada em consideração.

Uma situação em que se pode trabalhar com séries em níveis, evitando regressões espúrias ocorre quando as séries são co-integradas.

Johnston e Dinardo (1997, p. 301) expressam que, quando as variáveis no modelo VAR são integradas de primeira ordem ou mais, estão sujeitas às inconsistências de regressões, considerando que as variáveis são não estacionárias. A presença de variáveis não estacionárias, no entanto, cria a possibilidade de relações de co-integração, gerando o que é conhecido na literatura como mecanismo de correção de erros.

\subsubsection{Testes de Co-integração}

\subsubsection{Teste de Johansen}

Retoma-se a equação (3.10) do modelo VAR, em notação matricial, no entanto, agora considerando que $Y_{t}$ seja um vetor com $n$ variáveis ( $n \times 1), n \geq 2$, supondo que são integrados de ordem $1, I(1)$, não estacionárias. $\mathrm{O}$ vetor pode ser expresso por:

$$
Y_{t}=\alpha+\Pi_{1} Y_{t-1}+\Pi_{2} Y_{t-2}+\ldots+\Pi_{p} Y_{t-p}+\varepsilon_{t}
$$

A equação (3.10) pode ser modificada em termos de um Modelo de Correção de Erros, cujo formato é:

$$
\Delta Y_{t}=\Pi Y_{t-1}+\Gamma_{1} \Delta Y_{t-1}+\ldots+\Gamma_{p-1} \Delta Y_{t-p+1}+\varepsilon_{t}
$$

em que

$$
\begin{aligned}
& \Pi=\Pi_{1}+\Pi_{2}+\ldots+\Pi_{p}-I \quad i=1,2,3, \ldots, p-1 \\
& \Gamma_{i}=-\sum_{j=i+1}^{p} \Pi_{j} \\
& I=\text { matriz identidade }
\end{aligned}
$$


A matriz $\Pi(n \times n)$ pode ser vista com maior nível de detalhe, sendo representada por:

$$
\Pi=\alpha \beta^{\prime}
$$

em que

$\alpha$ : matriz que representa a velocidade de ajustamento dos parâmetros no curto prazo;

$\beta$ : matriz de coeficiente de co-integração de longo prazo, entre as variáveis.

Os parâmetros $\alpha$ e $\beta$ são matrizes de dimensão $n \times r$, em que $n$ é o número de variáveis incluídas no modelo e $r$ é o número de vetores de co-integração da matriz $\Pi$. O comportamento de $Y_{t}$ vai depender dos autovalores da matriz de longo prazo $\Pi$.

Pode-se ter então os seguintes casos (PATTERSON, 2000, p. 620; VALLS, 2004, p. 34):

i) se todos os autovalores de $\Pi$ são diferentes de zero (isto é, $r=n$, colunas linearmente independentes) e, portanto, esta matriz tem posto completo, $\Pi(1)=\Pi_{1}+\ldots+\Pi_{p}$ são menores do que 1 , implicando que todos os componentes de $Y_{t}$ são estacionários e a representação válida é o VAR (p) em nível dado por (3.10), não cabendo qualquer análise de co-integração;

ii) se todos os autovalores de $\Pi$ são zero (isto é, $r=0$ ), esta matriz é, portanto, indistinguível da matriz nula. Implica também que П(1) tem todos os autovalores iguais a um e, portanto, os componentes de $Y_{t}$ são no mínimo $I(1)$ e a representação válida é um VAR (p1) em primeira diferença, isto é, (3.11) sem o termo em nível. Tal formulação, todavia, não prevê nenhuma informação de relacionamento entre as variáveis no VAR, constituindo-se numa desvantagem, já que é neste aspecto que a Teoria Econômica é informativa. Esta opção, portanto, geralmente não é satisfatória, apesar do VAR em diferenças transformar os dados em estacionários e ser aceitável do ponto de vista estatístico;

iii) se $\Pi$ tem posto reduzido, isto é, $0<r<n$, neste caso tem-se $n-r$ autovalores diferentes de zero. Os componentes de $Y_{t}$ são no mínimo $I(1)$ e a representação válida é (3.11) com $\Pi=\alpha \beta^{\prime}$, onde $\alpha$ e $\beta$ são matrizes $n \times r$ de posto $r$. Esta representação é chamada 
Vetor de Correção de Erros (VEC) e nela estão presentes $r$ relações de co-integração.

Johansen e Juselius (1990) desenvolveram dois testes capazes de determinar o posto da matriz $\Pi$ da equação (3.12). O primeiro teste, conhecido como teste traço, é dado por:

$$
\lambda_{\text {trace }}=-T \sum_{i=r+1}^{n} \ln (1-\hat{\lambda}) \quad r=0,1,2, \ldots, n-2, n-1
$$

em que

$\hat{\lambda}$ : valor estimado dos autovalores obtidos da matriz $\beta$;

$T$ : número de observações.

O teste traço avalia a hipótese nula de que o número de vetores diferentes de co-integração é menor ou igual a $r$ contra uma hipótese geral.

$$
H_{o}: \lambda=0 \quad i=r+1, \ldots, n
$$

A não-rejeição de $H_{o}$ indica a presença de no máximo $r$ vetores de co-integração. Se $H_{o}$ for rejeitada deve-se repetir o teste para $r+1$ e determinar se existem $r+1$ vetores de co-integração.

O segundo teste é o teste do máximo autovalor que testa a existência de exatamente $r$ vetores de co-integração contra a alternativa de existência de $r+1$ vetores.

$$
\lambda_{\max }=-T \ln \left(1-\hat{\lambda}_{r+1}\right)
$$

Com a hipótese nula dada por:

$$
H_{o}: \lambda_{r+1}=0
$$

A não-rejeição de $H_{o}$ indica presença de exatamente $r$ vetores de co-integração.

A inclusão de termos deterministas também é essencial para correta implementação do procedimento de Johansen. Pode-se representar a inclusão destes termos em (3.11) por:

$$
\Delta Y_{t}=\Pi Y_{t-1}+\Gamma_{1} \Delta Y_{t-1}+\ldots+\Gamma_{p-1} \Delta Y_{t-p+1}+\Phi D_{t}+\varepsilon_{t}
$$


em que $D_{t}$ pode representar tanto uma constante, uma tendência e/ou uma variável dummy. A escolha dos termos deterministas deve ser feita com o auxílio de uma inspeção visual nos dados e também mediante testes apropriados sobre a significância dos termos deterministas. A determinação correta do número de defasagens é fundamental para análise de co-integração. A determinação do número de defasagens, ou seja, o valor de $p$ em (3.15) pode ser feita por vários métodos, entre eles, Akaike Information Criterion (1974), Schwarz (1978), e o de Hannan-Quinn (1979).

\section{Resultados e discussão}

Conforme comentado no item 3.2.1, uma ferramenta muito importante para análise de estacionariedade é o exame dos correlogramas das séries, baseados na Função de Autocorrelação (FAC). Assim, a inspeção visual dessas funções é o primeiro indicador de raiz unitária.

Foram inicialmente obtidos os correlogramas das séries de preços em níveis logaritmizadas. Observou-se que os coeficientes de autocorrelação iniciam com valores entre 0,77 (LFOR) e 0,92 (LBRA) e decaem lentamente com o aumento do número de defasagens $k$, variando entre 3 defasagens (LFOR), com o coeficiente de 0,47 e 5 defasagens (LSAL) com o coeficiente de autocorrelação de 0,57 , forte indício de presença de raiz unitária. Verificou-se também que as observações para todas as séries são positivamente correlacionadas, assinalando expressiva dependência dos valores presentes com os valores passados, o que caracteriza um modelo do tipo autor-regressivo, indicando também a não-estacionariedade das séries.

Os correlogramas obtidos na primeira diferença mostraram um padrão diferente. Os coeficientes de autocorrelação das séries diminuem acentuadamente após uma defasagem, oscilando em torno de zero, o que sugere ausência de dependência dos valores correntes com os valores passados, indicando a ausência de raiz unitária, ou seja, estacionariedade das séries. Deste modo, as séries devem ser $I$ (1) - integradas de ordem um.

O passo seguinte foi realizar o teste de raiz unitária. Para tanto, aplicou-se o Dickey-Fuller Aumentado (ADF) para verificar a estacio- 
nariedade das séries, com defasagens baseadas no AIC (Akaike Information Criterion), obtendo-se os resultados apresentados na Tabela 1. As estatísticas $\tau_{t}, \tau_{\mu}, \tau$ correspondem, respectivamente, às equações com constante e com tendência; com constante e sem tendência; e sem constante e sem tendência.

Tabela 1. Teste de Raiz Unitária, Dickey-Fuller Aumentado (ADF) para as séries de preço em níveis logarítmizadas, janeiro de 2001 a dezembro de 2005.

\begin{tabular}{lcccccc}
\hline & $\tau_{t}$ & defasagens & $\tau_{\mu}$ & defasagens & $\tau$ & defasagens \\
\hline LSP & $-4,3633^{*}$ & 3 & $-5,6292^{*}$ & 5 & $-3,6502^{*}$ & 2 \\
LNAT & $-4,4476^{*}$ & 0 & $-4,0220^{*}$ & 0 & $-2,6867^{*}$ & 0 \\
LFOR & $-4,5736^{*}$ & 1 & $-4,5200^{*}$ & 1 & $-3,4636^{*}$ & 1 \\
LSAL & $-4,0669^{*}$ & 9 & $-2,4780$ & 9 & $-2,0725^{* *}$ & 9 \\
LREC & $-3,1700^{* *}$ & 12 & $-2,7833^{* * *}$ & 12 & $-1,3043$ & 12 \\
LCUR & $-5,3890^{*}$ & 5 & $-4,2027^{*}$ & 0 & $-3,6745^{*}$ & 0 \\
LBH & $-5,3114^{*}$ & 2 & $-4,5302^{*}$ & 2 & $-3,9028^{*}$ & 2 \\
LBRA & $-6,4507^{*}$ & 4 & $-5,0765^{*}$ & 4 & $-3,1124^{*}$ & 2 \\
\hline
\end{tabular}

Fonte: Dados da pesquisa.

***indica que a hipótese nula é rejeitada ao nível de significância de $10 \%$.

**indica que a hipótese nula é rejeitada ao nível de significância de $5 \%$.

*indica que a hipótese nula é rejeitada ao nível de significância de $1 \%$.

Verificou-se que as séries LSP, LNAT, LFOR, LCUR, LBH e LBRA mostraram ser estacionárias ao nível de $1 \%$ de significância para os três modelos de equações. A série LSAL mostrou ser estacionária ao nível de $1 \%$ e ao nível de $5 \%$, respectivamente, para as equações com constante e com tendência e para a equação sem constante e sem tendência. No entanto, para a equação com constante e sem tendência não se rejeitou a hipótese nula de raiz unitária. A série LREC apresentou ser estacionária ao nível de $10 \%$ para as equações com constante e com tendência e com constante e sem tendência, porém para a equação sem constante e sem tendência não se rejeitou a hipótese nula de raiz unitária.

Dado que a maioria das séries em Economia possui raiz unitária, e visto que foram detectadas quebras estruturais, foram realizados testes de raiz unitárias mais adequados diante da presença destas quebras nas séries. Uma vez que o teste ADF é muito sensível à presença de valores 
atípicos, fez-se necessária a estimação dos testes de raiz unitária com presença de quebras. Na Tabela 2 são apresentados os resultados dos testes de estacionariedade formulados por Kwiatkowsk et al. (1992) - KPSS.

Tabela 2. Teste de Estacionariedade, Kwiatkowski-Phillips-Schmidt-Shin para as séries de preço em níveis logarítmizadas, janeiro de 2001 a dezembro de 2005.

\begin{tabular}{lcccc}
\hline & Tendência e Constante & defasagens & Constante & defasagens \\
\hline LSP & $0,1456^{* * *}$ & 11 & $1,1388^{*}$ & 11 \\
LNAT & 0,1025 & 11 & $0,8632^{*}$ & 11 \\
LFOR & 0,0444 & 11 & 0,1592 & 11 \\
LSAL & 0,0981 & 10 & $1,5748^{*}$ & 11 \\
LREC & 0,0527 & 10 & $0,8621^{*}$ & 11 \\
LCUR & $0,1946^{* *}$ & 10 & $1,4691^{*}$ & 11 \\
LBH & $0,1236^{* * *}$ & 11 & $1,0922^{*}$ & 11 \\
LBRA & 0,0576 & 10 & $1,1167^{*}$ & 11 \\
\hline
\end{tabular}

Fonte: Dados da pesquisa.

***indica que a hipótese nula é rejeitada ao nível de significância de $10 \%$.

**indica que a hipótese nula é rejeitada ao nível de significância de $5 \%$.

*indica que a hipótese nula é rejeitada ao nível de significância de $1 \%$.

As séries LNAT, LSAL, LREC e LBRA mostraram ser estacionárias com tendência e com constante, no entanto, com constante e sem tendência, as séries sinalizaram no sentido da rejeição da hipótese nula ao nível de $1 \%$ de significância. Foi rejeitada a hipótese nula de estacionariedade ao nível de $10 \%$ e $1 \%$, respectivamente, para os modelos com constante e com tendência e com constante e sem tendência, para as séries LSP e LBH. A série LCUR apresentou ser não estacionária ao nível de $5 \%$ de significância para o modelo com tendência e com constante e a hipótese nula de estacionariedade foi rejeitada ao nível de 1\% para o modelo com constante.

Portanto realizou-se o teste KPSS para as séries na primeira diferença. Os resultados estão apresentados na Tabela 3.

Todas as séries foram estacionárias na primeira diferença para os modelos de equações. Portanto, como os gráficos das séries apresentaram várias oscilações e, admitindo que os correlogramas de todas as séries em níveis têm certa memória, caracterizando assim a não-estacionariedade das séries, considerou-se que as variáveis são estacionárias 
nas diferenças. Desta maneira, podem ser consideradas como sendo integradas de ordem um.

Tabela 3. Teste de Estacionariedade, Kwiatkowski-Phillips-Schmidt-Shin para as séries de preço logarítmizadas na primeira diferença, janeiro de 2001 a dezembro de 2005.

\begin{tabular}{lcccc}
\hline & Tendência e Constante & defasagens & Constante & defasagens \\
\hline $\mathrm{D}($ LSP $)$ & 0,0133 & 4 & 0,0196 & 4 \\
$\mathrm{D}($ LNAT $)$ & 0,0206 & 9 & 0,0313 & 9 \\
$\mathrm{D}($ LFOR $)$ & 0,0222 & 7 & 0,0233 & 7 \\
$\mathrm{D}(\mathrm{LSAL})$ & 0,0102 & 1 & 0,0111 & 1 \\
$\mathrm{D}(\mathrm{LREC})$ & 0,0130 & 1 & 0,0250 & 1 \\
$\mathrm{D}(\mathrm{LCUR})$ & 0,0132 & 6 & 0,0167 & 6 \\
$\mathrm{D}(\mathrm{LBH})$ & 0,0128 & 3 & 0,0201 & 3 \\
$\mathrm{D}($ LBRA $)$ & 0,0126 & 6 & 0,0148 & 6 \\
\hline
\end{tabular}

Fonte: Dados da pesquisa.

Os valores críticos para o modelo com constante e com tendência ao nível de $1 \%, 5 \%$, e $10 \%$ são respectivamente $0,2160,0,1460$ e 0,1190 e para o modelo com constante e sem tendência os Valores Críticos são, ao nível de $1 \%(0,7390), 5 \%(0,4630)$ e $10 \%(0,3470)$. Portanto, não há rejeição da hipótese nula em nenhum dos níveis adotados.

Antes de realizar o teste de co-integração, foi necessário determinar o número de defasagens a serem utilizadas e escolher o modelo a empregar. O critério de informação usado para determinação do número de defasagens foi o de Akaike $(A I C)$. O critério de $A I C$ apresentou menor valor para defasagem de ordem dois, portanto, foram utilizadas, duas defasagens para realização do teste de Co-integração de Johansen.

O Critério de Schwarz apontou como melhor modelo o sem tendência determinística, mas com constante. Isto condiz com a análise gráfica das séries, pois percebe-se que há mudança de patamar indicando presença de intercepto, porém, não há um padrão condizente com tendência determinística.

Os resultados dos testes de co-integração apresentados na Tabela 4, mostram que a hipótese nula de não-co-integração foi rejeitada, uma vez que o valor calculado da estatística traço $\left(\boldsymbol{\lambda}_{\text {trace }}\right)$ é igual a 372,56 , o qual é superior ao seu respectivo valor crítico ao nível de $1 \%(177,20)$. Esse resultado indica que há pelo menos um vetor de co-integração, e o teste deve continuar até que a hipótese nula possa ser rejeitada. Portanto, concluiu-se que há 8 vetores de co-integração, visto que a 
hipótese nula de que existem até 7 vetores co-integrados foi rejeitada, pois o valor calculado $(11,73)$ para a estatística $\lambda_{\text {trace }}$ é superior ao seu respectivo valor crítico $(9,24)$, ao nível de $5 \%$.

Tabela 4. Resultado do Teste de Co-integração de Johansen, variáveis LSP, LNAT, LFOR, LSAL, LREC, LCUR, LBH e LBRA. Janeiro de 2001 a dezembro de 2005.

\begin{tabular}{lccccc}
\hline Eigenvalue & & & & & Estatística traço \\
& Nula & $\begin{array}{c}\text { Hipótese } \\
\text { Alter. }\end{array}$ & $\begin{array}{c}\text { Estatística traço } \\
\lambda_{\text {trace }} \text { calculado }\end{array}$ & $\begin{array}{c}\text { Estástica traço } \\
\lambda_{\text {trace }} \\
\text { ao nível de 5\% }\end{array}$ & $\begin{array}{c}\lambda_{\text {trace }} \text { valor } \\
\text { crítico ao nível } \\
\text { de } 1 \% .\end{array}$ \\
\hline 0,2821 & $r=0$ & $r>0$ & $372,5631^{*}$ & 165,80 & 177,20 \\
0,2692 & $r \leq 1$ & $r>1$ & $287,7049^{*}$ & 131,70 & 143,09 \\
0,2317 & $r \leq 2$ & $r>2$ & $207,3890^{*}$ & 102,14 & 111,01 \\
0,1488 & $r \leq 3$ & $r>3$ & $139,8999^{*}$ & 76,07 & 84,45 \\
0,1387 & $r \leq 4$ & $r>4$ & $98,6383^{*}$ & 53,12 & 60,16 \\
0,1035 & $r \leq 5$ & $r>5$ & $60,4068^{*}$ & 34,91 & 41,07 \\
0,0776 & $r \leq 6$ & $r>6$ & $32,4227^{*}$ & 19,96 & 24,60 \\
0,0448 & $r \leq 7$ & $r>7$ & $11,737^{* *}$ & 9,24 & 12,97 \\
\hline
\end{tabular}

Fonte: Dados da pesquisa.

**indica que a hipótese nula é rejeitada ao nível de significância de $5 \%$.

*indica que a hipótese nula é rejeitada ao nível de significância de $1 \%$.

Como o número de vetores de co-integração é igual ao número de variáveis, ou seja, o rank é pleno, deve-se utilizar o Modelo Vetorial Auto-Regressivo (VAR) em nível. Isso implica que, colocando todas as variáveis em nível no VAR, a combinação linear entre elas produz um relacionamento estacionário.

A Tabela 5 apresenta os resultados relativos à decomposição da variância dos erros de previsão para oito variáveis. A primeira coluna determina a variável atribuída a um choque não antecipado. A segunda coluna representa os períodos, no presente trabalho expressos em semanas. Considera-se também que um choque não antecipado sobre as variáveis analisadas perdure no máximo por 24 semanas. No caso da variável LSP, a terceira coluna informa o percentual da variância dos erros de previsão em função de choques não antecipados sobre essa variável, ou seja, mede qual o efeito que um choque não antecipado sobre LSP tem sobre ela mesma ao longo do tempo. As demais colunas 
captam os percentuais das variâncias dos erros de previsão de LSP atribuídos às variáveis em LNAT, LFOR, LSAL, LREC, LCUR, LBH e LBRA.

Os resultados da decomposição da variância dos erros de previsão de LSP mostraram que, decorridos 24 semanas após um choque não antecipado sobre essa variável, aproximadamente $76 \%$ de seu comportamento decorre dela própria, e aproximadamente $24 \%$ é atribuído às outras variáveis, as quais são: LNAT (0,52\%), LFOR (2.78\%), LSAL (1.03\%), LREC $(2,22 \%)$, LCUR $(6,82 \%)$, LBH $(4.10 \%)$ e LBRA $(6,37 \%)$.

Tabela 5. Decomposição da Variância dos Erros de Previsão em Percentagem de LSP para as variáveis LSP, LNAT, LFOR, LSAL, LREC, LCUR, LBH e LBRA. janeiro de 2001 a dezembro de 2005.

\begin{tabular}{cccccccccc}
\hline Variável & Período & LSP & LNAT & LFOR & LSAL & LREC & LCUR & LBH & LBRA \\
\hline LSP & 1 & 100,00 & 0,0000 & 0,0000 & 0,0000 & 0,0000 & 0,0000 & 0,0000 & 0,0000 \\
& 6 & 85,187 & 0,3917 & 0,1860 & 0,8946 & 1,5860 & 5,9607 & 4,6263 & 1,1667 \\
& 12 & 80,589 & 0,3408 & 1,4415 & 0,9375 & 1,5111 & 7,0776 & 4,2369 & 3,8646 \\
& 18 & 77,108 & 0,4556 & 2,4768 & 1,0185 & 2,0467 & 6,8942 & 4,1222 & 5,8776 \\
& 24 & 76,115 & 0,5280 & 2,7878 & 1,0389 & 2,2271 & 6,8222 & 4,1001 & 6,3796 \\
\hline
\end{tabular}

Fonte: Dados da Pesquisa.

Em relação à LNAT, Tabela 6, grande parte de seu próprio erro de previsão é explicada pelas variáveis externas, cerca de $68 \%$, decorridos 24 meses após um choque inicial não antecipado, no qual a maior parte da variância de LNAT deve-se a LSP $(51,07 \%)$. Em torno de $31 \%$ referese a ela própria.

Tabela 6. Decomposição da Variância dos Erros de Previsão em Percentagem de LNAT para as variáveis LSP, LNAT, LFOR, LSAL, LREC, LCUR, LBH e LBRA. janeiro de 2001 a dezembro de 2005.

\begin{tabular}{cccccccccc}
\hline Variável Período & LSP & LNAT & LFOR & LSAL & LREC & LCUR & LBH & LBRA \\
\hline LNAT & 1 & 1,4656 & 98,534 & 0,0000 & 0,0000 & 0,0000 & 0,0000 & 0,0000 & 0,0000 \\
& 6 & 37,273 & 54,120 & 0,0580 & 0,6302 & 1,9513 & 2,8584 & 2,9629 & 0,1447 \\
& 12 & 52,549 & 34,922 & 0,3960 & 0,4578 & 1,6161 & 4,9585 & 3,5060 & 1,5933 \\
& 18 & 51,877 & 31,977 & 1,3159 & 0,5382 & 1,8755 & 5,0463 & 3,4639 & 3,9057 \\
& 24 & 51,077 & 31,356 & 1,7439 & 0,5745 & 2,1042 & 4,9885 & 3,4451 & 4,7095 \\
\hline
\end{tabular}

Fonte: Dados da Pesquisa. 
Conforme Tabela 7 , em torno de $49 \%$ é a variância do seu erro de previsão da variável LFOR, decorridas 24 semanas após o choque inicial não antecipado. Os $51 \%$ restantes são atribuídos às demais variáveis, distribuídas da seguinte forma: LSP $(33,23 \%)$, LNAT $(7,41 \%)$, LSAL $(0,56 \%)$, LREC $(4,07 \%)$, LCUR $(0,91 \%), \operatorname{LBH}(2,64 \%)$ e LBRA $(1,79 \%)$.

Tabela 7. Decomposição da Variância dos Erros de Previsão em Percentagem de LFOR para as variáveis LSP, LNAT, LFOR, LSAL, LREC, LCUR, LBH e LBRA. Janeiro de 2001 a dezembro de 2005.

\begin{tabular}{cccccccccc}
\hline Variável Período & LSP & LNAT & LFOR & LSAL & LREC & LCUR & LBH & LBRA \\
\hline LFOR & 1 & 3,8865 & 9,2410 & 86,872 & 0,0000 & 0,0000 & 0,0000 & 0,0000 & 0,0000 \\
& 6 & 23,695 & 9,2107 & 59,552 & 0,5310 & 3,3748 & 0,1996 & 2,3025 & 1,1330 \\
& 12 & 32,717 & 7,5738 & 50,422 & 0,5636 & 4,0962 & 0,8447 & 2,6347 & 1,1463 \\
& 18 & 33,320 & 7,4273 & 49,469 & 0,5582 & 4,0436 & 0,9180 & 2,6422 & 1,6208 \\
& 24 & 33,237 & 7,4146 & 49,357 & 0,5619 & 4,0734 & 0,9171 & 2,6405 & 1,7969 \\
\hline
\end{tabular}

Fonte: Dados da Pesquisa.

Para a variável LSAL, Tabela 8, 24 semanas após um choque não antecipado sobre essa variável, apenas $28 \%$ da sua decomposição da variância dos erros de previsão decorre dela mesma, sendo os $72 \%$ restantes, das outras variáveis. Verificou-se, no entanto, que desses $72 \%$, LSP responde por cerca de $45 \%$.

Tabela 8. Decomposição da Variância dos Erros de Previsão em Percentagem de LSAL para as variáveis LSP, LNAT, LFOR, LSAL, LREC, LCUR, LBH e LBRA. janeiro de 2001 a dezembro de 2005.

\begin{tabular}{cccccccccc}
\hline Variável Período & LSP & LNAT & LFOR & LSAL & LREC & LCUR & LBH & LBRA \\
\hline LSAL & 1 & 7,3999 & 1,4241 & 2,8748 & 88,301 & 0,0000 & 0,0000 & 0,0000 & 0,0000 \\
& 6 & 40,078 & 0,7284 & 3,3130 & 43,676 & 0,4467 & 6,0386 & 4,3475 & 1,3706 \\
& 12 & 48,032 & 0,5198 & 4,4772 & 31,045 & 0,6868 & 8,7150 & 3,9615 & 2,5619 \\
18 & 46,534 & 0,5911 & 5,6454 & 28,669 & 1,4194 & 8,5536 & 3,8131 & 4,7725 \\
& 24 & 45,796 & 0,6726 & 6,0392 & 28,088 & 1,7007 & 8,4280 & 3,7820 & 5,4926 \\
\hline
\end{tabular}

Fonte: Dados da Pesquisa.

Conforme Tabela 9, os resultados da decomposição da variância dos erros de previsão de LREC mostraram que, decorridos 24 semanas após um choque não antecipado sobre essa variável, aproximadamente $45 \%$ de seu comportamento decorre dela própria, sendo que os aproximadamen- 
te $55 \%$ restantes, são atribuídos a LSP $(42,95 \%)$, LNAT $(0,81 \%)$, LFOR $(1,71 \%)$, LSAL $(0,92 \%)$, LCUR $(2,02 \%)$, LBH $(1,84 \%)$ e LBRA $(3,97 \%)$.

Tabela 9. Decomposição da Variância dos Erros de Previsão em Percentagem de LREC para as variáveis LSP, LNAT, LFOR, LSAL, LREC, LCUR, LBH e LBRA. Janeiro de 2001 a dezembro de 2005.

\begin{tabular}{cccccccccc}
\hline Variável Período & LSP & LNAT & LFOR & LSAL & LREC & LCUR & LBH & LBRA \\
\hline LREC & 1 & 1,5111 & 0,5275 & 0,4593 & 0,2605 & 97,241 & 0,0000 & 0,0000 & 0,0000 \\
& 6 & 36,171 & 0,8375 & 1,2221 & 0,9214 & 55,610 & 0,6179 & 1,6573 & 2,9608 \\
& 12 & 43,502 & 0,7928 & 1,1811 & 0,8690 & 46,946 & 1,9473 & 1,8541 & 2,9064 \\
& 18 & 43,186 & 0,7888 & 1,5749 & 0,9101 & 45,914 & 2,0319 & 1,8499 & 3,7429 \\
& 24 & 42,956 & 0,8122 & 1,7170 & 0,9206 & 45,739 & 2,0264 & 1,8495 & 3,9771 \\
\hline
\end{tabular}

Fonte: Dados da Pesquisa.

Em relação à variável LCUR, um choque não antecipado sobre essa variável representa em torno de $31 \%$ de seu comportamento, após 24 semanas. A maior parte (69\%), porém, deve-se às variáveis externas, sendo que a variável LSP representa $50 \%$ da variância do erro de previsão (Tabela 10).

Tabela 10. Decomposição da Variância dos Erros de Previsão em Percentagem de LCUR para as variáveis LSP, LNAT, LFOR, LSAL, LREC, LCUR, LBH e LBRA. janeiro de 2001 a dezembro de 2005.

\begin{tabular}{cccccccccc}
\hline Variável & Período & LSP & LNAT & LFOR & LSAL & LREC & LCUR & LBH & LBRA \\
\hline LCUR & 1 & 3,9438 & 1,0927 & 0,0159 & 0,4218 & 0,3069 & 94,218 & 0,0000 & 0,0000 \\
& 6 & 50,673 & 0,6890 & 0,7186 & 2,7656 & 0,2320 & 42,635 & 1,8998 & 0,3852 \\
& 12 & 53,607 & 0,5886 & 2,6375 & 2,5600 & 0,8636 & 33,873 & 2,2957 & 3,5728 \\
& 18 & 51,465 & 0,7133 & 3,8559 & 2,5512 & 1,5866 & 31,687 & 2,3070 & 5,8334 \\
& 24 & 50,730 & 0,7946 & 4,2244 & 2,5486 & 1,8192 & 31,116 & 2,3150 & 6,4507 \\
\hline
\end{tabular}

Fonte: Dados da Pesquisa.

Para variável LBH (Tabela 11), o grupo de variáveis externas é o principal responsável pela variância do seu erro de previsão, em torno de $82 \%$, tendo a seguinte distribuição: LSP $(63,25 \%)$, LNAT $(0,32 \%)$, LFOR $(2,95 \%)$, LSAL (1,29\%), LREC (1,94\%), LCUR $(7,04 \%)$ e LBRA (6,04\%). Observe-se que, após 24 meses, a maior parte da variância de LBH decorre de LSP. 
Tabela 11. Decomposição da Variância dos Erros de Previsão em Percentagem de LBH para as variáveis LSP, LNAT, LFOR, LSAL, LREC, LCUR, LBH e LBRA. Janeiro de 2001 a dezembro de 2005.

\begin{tabular}{cccccccccc}
\hline Variável Período & LSP & LNAT & LFOR & LSAL & LREC & LCUR & LBH & LBRA \\
\hline LBH & 1 & 19,363 & 0,0902 & 0,5422 & 0,1498 & 0,0001 & 0,5084 & 79,346 & 0,0000 \\
& 6 & 65,905 & 0,1018 & 0,7791 & 1,2933 & 1,2293 & 6,2070 & 23,661 & 0,8228 \\
& 12 & 66,599 & 0,1214 & 1,6688 & 1,2226 & 1,2247 & 7,3151 & 18,363 & 3,4845 \\
& 18 & 64,041 & 0,2464 & 2,6492 & 1,2816 & 1,7637 & 7,1215 & 17,384 & 5,5115 \\
& 24 & 63,251 & 0,3214 & 2,9539 & 1,2976 & 1,9472 & 7,0458 & 17,161 & 6,0208 \\
\hline
\end{tabular}

Fonte: Dados da Pesquisa.

A variável LBRA apresenta um quadro semelhante à variável LBH, pois a maior parte do erro de previsão é explicada pelas outras variáveis (cerca de $78 \%$ ), e a maior parte da variância $(57,56 \%)$ também é decorrente de LSP (Tabela 12).

Tabela 12. Decomposição da Variância dos Erros de Previsão em Percentagem de LBRA para as variáveis LSP, LNAT, LFOR, LSAL, LREC, LCUR, LBH e LBRA. Janeiro de 2001 a dezembro de 2005.

\begin{tabular}{cccccccccc}
\hline Variável Período & LSP & LNAT & LFOR & LSAL & LREC & LCUR & LBH & LBRA \\
\hline LBRA & 1 & 19,336 & 0,1282 & 0,0594 & 0,1536 & 0,8131 & 8,0338 & 2,1058 & 69,368 \\
& 6 & 55,760 & 0,1196 & 0,5100 & 0,5295 & 2,8921 & 12,062 & 2,8625 & 25,263 \\
& 12 & 59,967 & 0,1527 & 1,1121 & 0,6419 & 2,4206 & 12,742 & 2,6398 & 20,322 \\
& 18 & 58,171 & 0,2102 & 2,0011 & 0,7425 & 2,8508 & 12,360 & 2,5969 & 21,066 \\
& 24 & 57,560 & 0,2688 & 2,2766 & 0,7653 & 3,0096 & 12,231 & 2,5921 & 21,295 \\
\hline
\end{tabular}

Fonte: Dados da Pesquisa.

Como ilustrado na Figura 1, um choque não antecipado sobre os preços de melão amarelo, no contexto de atacado de LNAT e LFOR, permite observar que, apesar de as CEASAS de Natal e Fortaleza estarem localizadas dentro da área de influência dos dois maiores pólos de produção de melão, não exercem influência significativa sobre o comportamento dos preços das outras CEASAS analisadas. Isso ocorre, possivelmente, em razão de que a maior parte do melão amarelo comercializado no mercado interno destina-se ao Centro-Sul do país, principalmente para São Paulo. 
Sabe-se que os pólos Rio Grande do Norte e Ceará são responsáveis por $78 \%$ da produção nacional, e que $70 \%$ dessa produção é encaixada e o restante é vendido a granel. A metade da produção encaixada é destinada à exportação e da outra metade encaixada, cerca de $97 \%$ vai para os mercados localizados no Centro-Sul do país (Sudeste, 70\%; Centro Oeste, $15 \%$ e Sul, $12 \%$ ). O mercado nacional é representado, principalmente, pelos grandes centros consumidores (São Paulo, Rio de Janeiro, Belo Horizonte e Brasília). Tais centros de consumo estão se organizando nos moldes dos grandes mercados internacionais de produtos hortícolas, exigindo frutos de alta qualidade embalados em caixas. Os $3 \%$ restantes (da produção encaixada comercializada internamente) são consumidos em nichos de mercados das regiões Norte e Nordeste. Todo o melão a granel (30\% da produção total), geralmente de qualidade inferior, é comercializado nos mercados local e regional.

Com relação à Figura 2, um choque não antecipado sobre os preços de São Paulo induz uma rápida elevação dos preços de atacado das CEASAS de Natal, Salvador, Recife, Curitiba, Belo Horizonte e Brasília. Essa elevação de preço ocorre entre a quarta e a sexta semanas e, a partir desse ponto, começa a decair lentamente, até estabilizar-se entre o vigésimo e vigésimo quarto períodos. Os efeitos de aumentos de preços de melão de São Paulo no atacado tendem a ser absorvidos pelos outros mercados atacadistas ao longo do tempo até esgotarem-se, ao final de 24 semanas após esse choque inicial.

Verificou-se que um choque não antecipado sobre os preços de melão amarelo no atacado em São Paulo tem um impacto bem definido sobre os demais mercados atacadistas (as CEASAS estudadas). Isso provavelmente acontece porque é na CEASA de São Paulo onde ocorre maior concentração de volume comercializado no mercado brasileiro de melão, exercendo o papel de centro distribuidor para os outros mercados atacadistas, sendo a maior intermediadora do país.

Isso possivelmente ocorre em conseqüência da queda da quantidade ofertada de melão amarelo, e o decorrente aumento nos preços; e também das imperfeições do mercado distribuidor de melão, formado por atacadistas operando oligopolisticamente, em razão da grande quantidade de melão de que dispõem os atacadistas de São Paulo. Estes atacadistas distribuem um melão de melhor qualidade para os merca- 


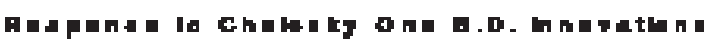

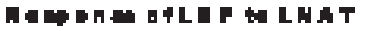

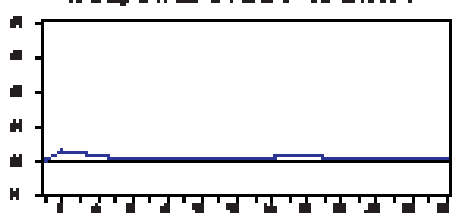

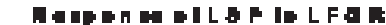
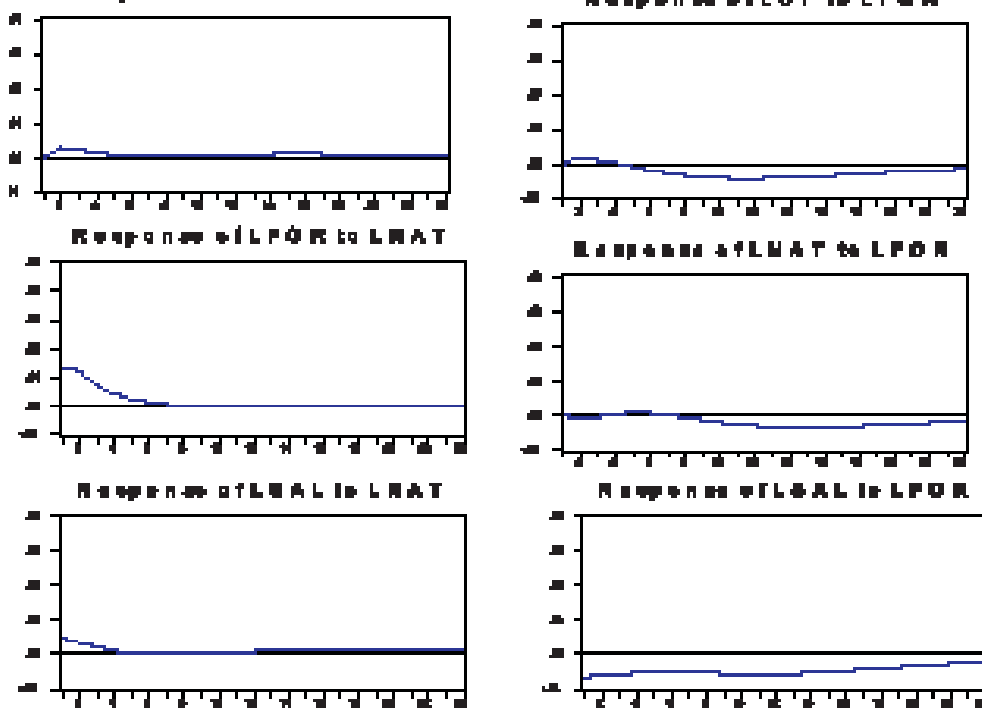

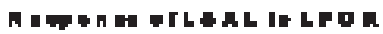
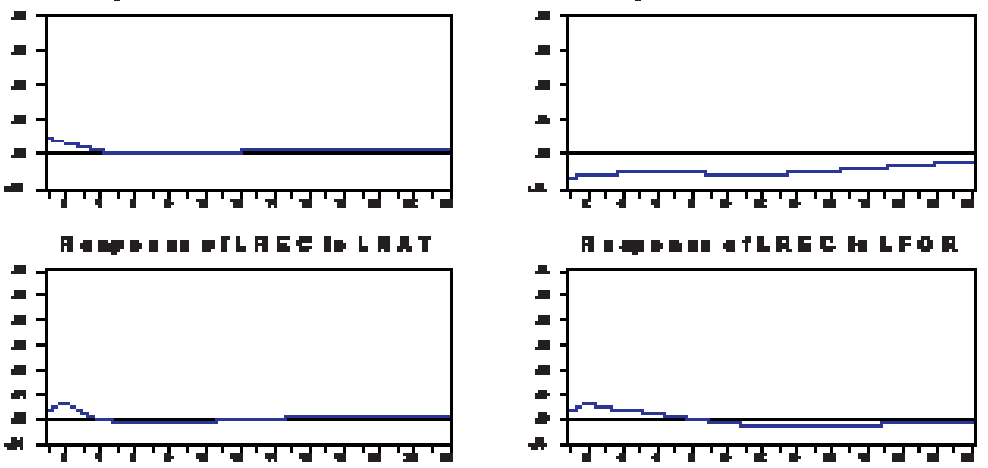

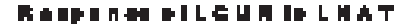
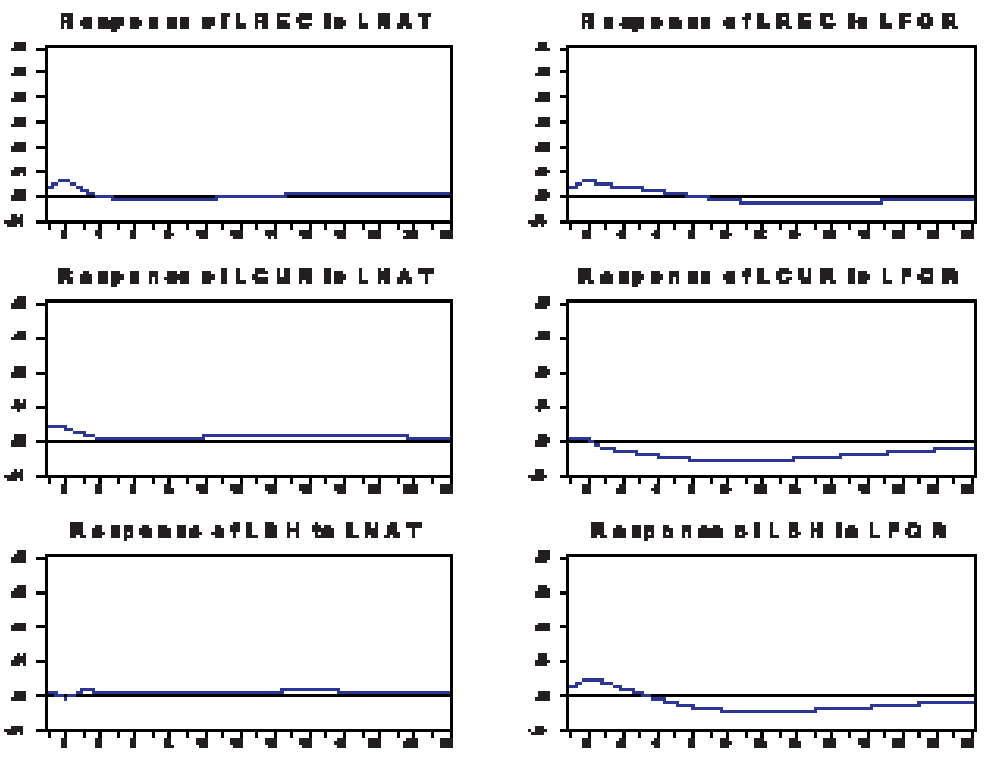

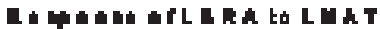

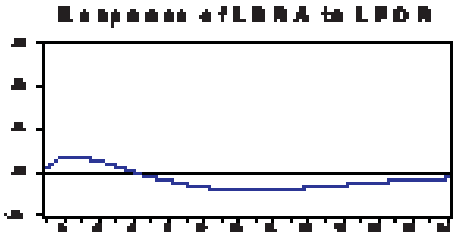

RER, Rio de Janeiro, vol. 45, no 03, p. 675-704, jul/set 2007 - Impressa em agosto 2007 
dos de Salvador, Recife, Curitiba, Belo Horizonte e Brasília, e também para Natal e Fortaleza, capitais dos maiores produtores nacionais, Rio Grande do Norte e Ceará, respectivamente.

Portanto, estes mercados aparecem como tomadores de preços, ou seja, dependem das decisões tomadas pelos atacadistas nacionais, representados principalmente pelos atacadistas de São Paulo, confirmando que a Companhia de Entrepostos e Armazéns Gerais de São Paulo (CEAGESP) serve como termômetro do mercado para os produtores e atacadistas.

\section{Conclusões e sugestões}

Este trabalho analisou o comportamento dos preços, no atacado, do melão amarelo produzido e comercializado no Brasil durante o período de janeiro de 2001 a dezembro de 2005, a partir da utilização de métodos relacionados ao campo de séries de tempo. O resultado do teste de co-integração não rejeitou a hipótese de que as variáveis são co-integradas, isto é, de que existe um relacionamento de equilíbrio de longo prazo entre elas.

Verificou-se que, apesar de os pólos Açu/Mossoró-RN e Baixo Jaguaribe-CE representarem as maiores áreas produtoras nacionais de melão, um choque não antecipado nos preços das Centrais de Abastecimento de Natal e Fortaleza não afetam de forma significativa os preços dos mercados atacadistas estudados. Constatou-se, no entanto, que é o mercado atacadista de São Paulo que influencia o comportamento dos preços nas Centrais de Abastecimento de Natal-RN, Fortaleza-CE, Recife-PE, Salvador-BA, Curitiba-PR, Belo Horizonte - MG e Brasília-DF.

Uma variação nos preços agrícolas, no atacado em São Paulo, portanto, tem impacto bem definido sobre os demais mercados atacadistas, citados anteriormente. Isso possivelmente acontece porque é na Companhia de Entrepostos e Armazéns Gerais de São Paulo (CEAGESP) onde ocorre maior concentração de volume comercializado no mercado brasileiro de melão, desempenhando o papel de centro distribuidor para os outros mercados atacadistas, sendo a maior intermediadora do país.

Assim sendo, as CEASAS brasileiras que comercializam melão aparecem como tomadoras de preços, ou seja, dependem das decisões tomadas pelos atacadistas da CEAGESP, constituindo, na prática, um mer- 
cado oligopolístico de melão, com epicentro na CEAGESP. Estes atacadistas impõem preços no mercado e as outras centrais de abastecimento brasileiras comportam-se como tomadores de preços.

Para limitar as imperfeições do mercado do melão no Brasil, é preciso que os produtores criem uma estrutura de chegada a São Paulo, ou uma logística de comercialização própria que os faça menos dependentes das decisões tomadas pelos atacadistas de São Paulo.

Sugere-se a criação de uma cooperativa que represente os pequenos, médios e grandes produtores, que, provavelmente, permitirá aumentar tanto seu poder de barganha como seu poder financeiro, viabilizando a implantação de uma infra-estrutura de chegada adequada à São Paulo. Pode-se assim, diminuir custos de comercialização, aumentar os lucros, possibilitar melhores preços para os consumidores e diminuir as imperfeições de mercado. Simultaneamente, deve-se garantir a qualidade do melão produzido e comercializado pela cooperativa, mediante a criação de selo de garantia.

\section{Referências bibliográficas}

AGRIANUAL 2005. Anuário da Agricultura Brasileira. FNP - Consultoria e Comércio. São Paulo-SP, 2005.

ARDENI, P.G. Does the law of one price really hold? American Journal of Agricultural Economics, V. 71, N. 3, p. 661-669, Aug. 1989.

AKAIKE, H. A new look at the statistical model identification. IEEE Transaction on Automatic Control. AC-19 (6), p. 716-723. 1974.

BISHOP, R.V. The Construction and Use of Causality Test. Agricultural and Economics Research, V. 31, N. 4, p. 1-6, Oct. 1979.

BRASIL. MINISTÉRIO DE DESENVOLVIMENTO INDUSTRIA E COMÉRCIO - MDIC - ALICEWeb. Valores e quantidades exportadas no mundo: 2000 a 2004. Brasília-DF. Disponível em: < aliceweb.desenvolvimento.gov.br > . Acesso em: 11 nov. 2005.

COELHO, A.B. A cultura do Algodão e a questão da Integração entre preços internos e externos. 2002. 136f. Dissertação (Mestrado em Economia) - Departamento de Economia da Faculdade de Economia, Administração e Contabilidade da Universidade de São Paulo, São Paulo, 2002. 
DICKEY, D.A.; FULLER, W.A. Distribution of the estimators for autoregressive time series with a unit root. Journal of American Statistical Association, V. 74, N. 366, p. 427-431, Jun. 1997.

Likelihood ratio statistics for autoregressive time series with a unit root. Econometrica, V. 49, N. 4, p. 1057-1072, Jul. 1981.

ENDERS, W. Applied econometric time series. New York: John Wiley and Sons, 1995.

FERNANDES, M.; TORO, J. O Mecanismo Monetário de transmissão na Economia Brasileira Pós-Plano Real. Revista Brasileira de Economia, V. 59, N. 1, p. 5-32, Jan./Mar. 2005. Disponível em: < epge.fgv.br/portal/arquivo/1293.pdf > Acesso em: 15 jun. 2006.

FRUTAS DO BRASIL. Melão - Produção. Empresa Brasileira de Pesquisa Agropecuária. Embrapa Agroindústria Tropical. Ministério da Agricultura e do Abastecimento. Brasília-DF. 2003.

FRUTISÉRIES 2. Ceará - Melão. Ministério da Integração Nacional - MI. Secretaria de Infra-Estrutura Hídrica - SIH. Brasília-DF: Departamento de Desenvolvimento Hidroagrícola - DDH. 2003.

GOLETTI, F.; RAISUDDIN, A.; FARID, N. Structural determinants of market integration. The case of rice markets in Bangladesh. The Developing Economies, V. 33, N. 2, p. 185-202, Jun. 1995.

GRANGER, C.W.J. Investigating causal relationship by econometric models and cross-spectral methods. Econometrica, V. 37, N. 3, p. 424-439. Jul. 1969.

GRANGER, C.W.J.; NEWBOLD, P. Spurious Regressions in Econometrics. Journal of Econometrics. N. 2, p. 111-120. 1974.

GREENE, W.H. Econometric analysis. 4 ed. New Jersey: Prentice Hall. 2000.

GUJARATI, D.N. Econometria básica. 3 ed. São Paulo: Makron Books, 2000.

HANNAN, E.I.; QUINN, B.G. The determination of the order of an autoregression. Journal of the Royal Statistic Society. B, 41, p. 190-195. 
JOHANSEN, S.; JUSELIUS, K. Maximum Likelihood estimation and inference on cointegration with applications to the demand for money. Oxford Bulletin of Economics and Statistics, V. 52, N. 2, p. 169-210, 1990.

JOHNSTON, J.; DINARDO, J. Econometric Methods. 4 ed. Singapore: McGraw-Hill. 1997.

KWIATKOWSKI, D. et al. Testing the null hypothesis of stationarity against the alternative of a unit root. How sure are we that economic time series have a unit root? North-Holland: Journal of Econometrics, V. 54, p. 159-178. 1992.

MARGARIDO, M.A. Transmissão de preços internacionais de suco de laranja para preços ao nível de produtor de laranja no Estado de São Paulo. Instituto de Economia Agrícola. Coleção Estudos Agrícolas 6. São Paulo-SP. 1998.

et al. Análise dos efeitos preço e câmbio sobre o preço do óleo de soja na Cidade de São Paulo: uma aplicação do modelo VAR. Revista de Economia e Sociologia Rural, Juiz de Fora-MG, V. 15, N. 1, p. 69106. 2002

MATOS, O.C. Econometria básica: teoria e aplicações. 3 ed. São PauloSP: Ed. Atlas. 2000.

MORENTTIN, P.A.; TOLOI, C.M.C. Análise de séries temporais. São Paulo-SP: Edgard Blücher. 2004.

PATTERSON, K.D. An Introduction to Applied Econometrics: a time series approach. Great Britain: St. Martin’s Press. 2000.

PINDYCK, R.S.; RUBINFELD, D.L. Econometria: Modelos e previsões. 4 ed. Rio de Janeiro: Ed. Campos. 2004.

RAVALLION, M. Testing marketing integration. American Journal of Agriculture Economics, V. 68, N. 1, p. 102-109, Feb. 1986.

SCHWARZ, G. Estimating the dimension of a model. Annals of Statistics. 6(2), p. 461-464.

SIMS, C.C. Money, Income, and Causality. American Economic Review, V. 62, N. 4, p. 540-555, Sep. 1972. 
VALLS, P. Introdução a séries temporais usando Eviews 4.1. São Paulo. Ibmec Business School. 2004.

ZAPATA, O.H.; GIL, J.M. Cointegration and causality in international agricultural economics research. Agricultural Economics, V. 20, N. 1, p. 1-9, Jan. 1999.

Recebido em janeiro de 2007 e revisto em março de 2007. 